Michael Svennevig

is Director of the Research Centre for Future

Communications at the

University of Leeds Institute of

Communications Studies, and

is a psychologist by training.

Before helping establish the

Centre in 1995, he worked at

senior level in the market

research industry and also ran

his own media research

company. In his early career he worked in the $B B C$ and the Independent Broadcasting Authority (IBA) research departments.

Keywords: interactive, television, uses of TV, TV programming, internet, survey research
Michael Svennevig

Research Centre for Future

Communications

University of Leeds

16 Clarendon Place

Leeds LS2 9JT, UK

Tel: +44 (0) 1133431604

Fax: +44 (0) 1133431117

E-mail: m.svennevig@leeds.ac.uk

\section{The interactive viewer: Reality or myth?}

\author{
Michael Svennevig \\ Received (in revised form): 29 June 2004
}

\begin{abstract}
The advent of interactive digital television (iTV) in the UK has the potential to revolutionise the use of the television set. But, as past technological innovations reveal, there may well be a great difference between the potential uses of a technology and the actual uses. The key issue is how people in the UK will use iTV in their everyday lives, and whether they will use the interactive capacities made available through iTV or whether they will use iTV as 'more television'. Available data suggest that the impact of iTV will be less dramatic than often predicted, and will be more of an evolutionary process than a revolutionary change. Interactivity per se may not be as powerful an influence on people's patterns of television use as has been predicted, compared with its core role in internet use.
\end{abstract}

\section{'New' and 'old' technologies}

The past and current interest in new and emerging communication devices generally centres around the core concept of information - for example, Manuel Castells' influential 'information technology revolution' and 'network society' concepts, proposing that information is becoming and will be a - if not the — primary commodity driving economic change and development. ${ }^{1}$

On offer are, or will be, home shopping, home banking and other financial services, internet and web access via the 'smart' TV, cinemaquality TV pictures, literally hundreds of new TV and radio channels, video on demand (VOD), electronic newspapers and e-books, 'mix-andmatch' TV channels and news, remote medical diagnosis, sharing virtualreality experiences with others, sending text, data, voice, streaming video, pictures to and from a range of fixed or mobile devices ... the list is seemingly endless, and possibly becoming overwhelming from the consumer point of view.

Certainly, many UK households have become 'technology-rich' in a short period of time and the majority of people have access to a wide range of media and delivery systems. Figure 1 shows the 2003 levels of domestic penetration of 23 of the key technologies tracked by the University of Leeds' futura.com longitudinal study of technological uptake and use (see Appendix). ${ }^{2}$ Only the more directly media-related technologies are shown - the study also tracks other technologies which have become increasingly widespread, such as multiple phone lines and answerphones.

What is also clear from these data is the important point that non- 


\section{Svennevig}

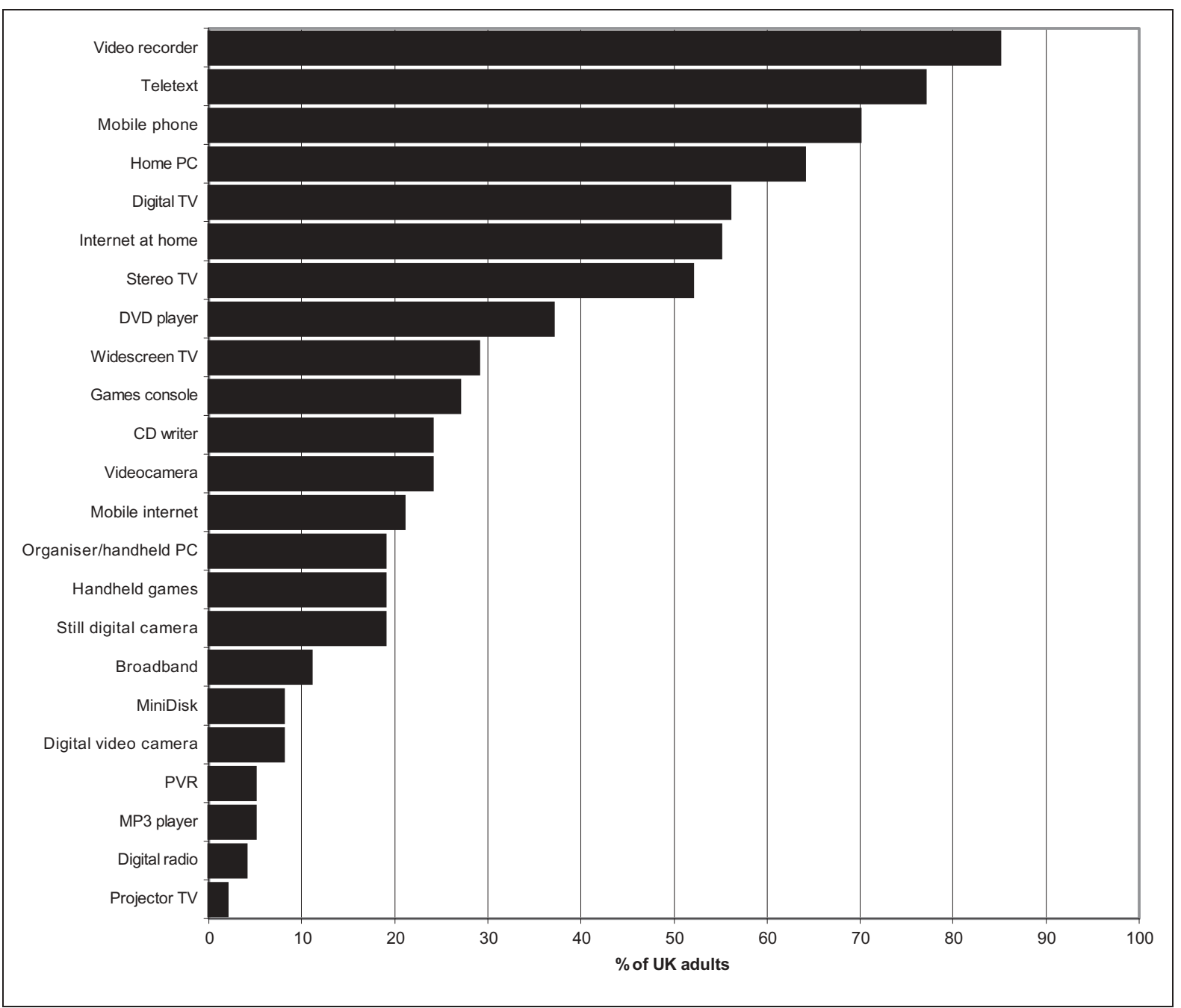

Figure 1: Technology in the UK home

\section{Old and new technologies co-exist}

digital ('old') and digital ('new') technologies happily co-exist in the average British home; a point often overlooked by some of the more enthusiastic proponents of the digital revolution. Old technologies are not jettisoned when a newer 'rival' turns up, any more than buying a new widescreen TV set implies throwing out its predecessor. Rather, the old and the new serve to increase choice within the home. Now the DVD player and the VCR can be used as appropriate, expanding flexibility and reducing dependence upon a single format.

Moreover, there are several items in the chart which did not exist or had barely reached the mass market in 1996, yet which are now commonplace: the mobile phone, the home PC and home internet, DVD players and stereo and widescreen TV. Basically, the typical UK home has become colonised by a wide range of technological applications - most of which, but not all, are digital. Many of these have shown very rapid growth since 1996 (shown in Figure 2). But not all have shown such rapid growth - internet access via TV or via mobiles (which is becoming more 


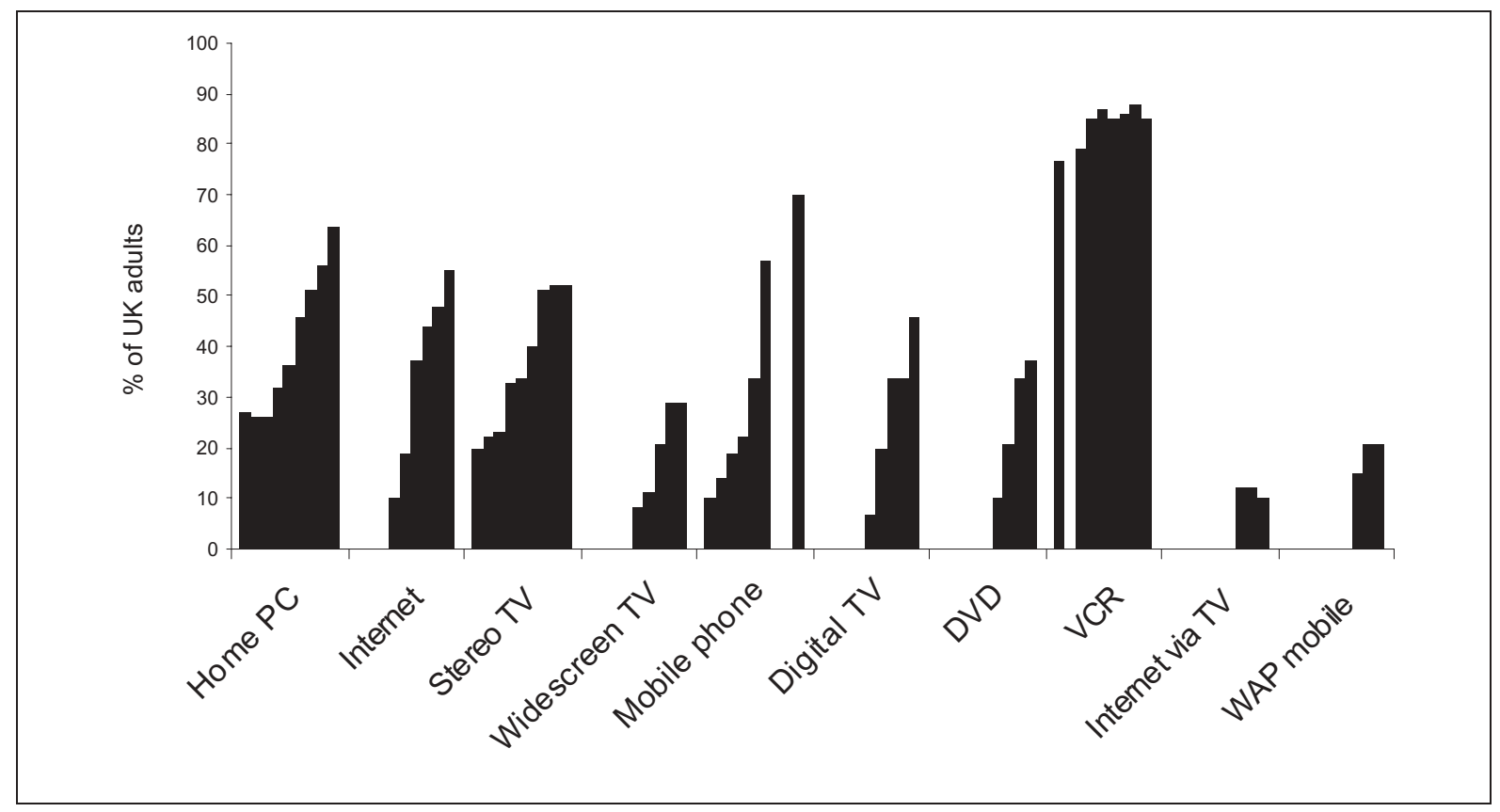

Figure 2: Technological growth over time, 1996-2003

\section{People are seeking benefits}

prevalent, mainly because it increasingly comes as standard when renting mobile phones) when compared to internet access via PC, for example.

The highest growth rates over the time period are for devices linked to the longer-established communications media: television and telephony, rather than for information-based technologies. For example, while the 'basic' mobile phone has shown striking levels of growth in terms of offering interpersonal communications (speech and text), its internetcapable possibilities appear to be distinctly less attractive to consumers as a whole.

People appear to be seeking ease of access to whatever it is they want - benefit-driven rather than medium-driven. So the VCR co-exists with the DVD player and the personal video recorder (PVR) (albeit still rarely found in the domestic setting): indeed people with VCRs are also the most likely to have DVD players and PVRs.

There is also evidence that the various benefits which derive from the various forms of technology are perceived by most people as being different. This helps explain why the locations of digital TV sets and PCs are quite different within the typical home. It also helps explain the continuing low uptake of 'combined' PC/TVs. The home PC tends not to be found in those rooms where members of a household generally gather together - living rooms, dining rooms, kitchens. In contrast, the typical $\mathrm{PC}$ is mainly placed in less frequently used parts of the home, whereas digital TV sets tend to found in the 'public' areas for shared use, and in bedrooms.

\section{Communication versus information}

In describing many of the benefits which are offered to the consumer by the new technologies, well-established terms such as 'communication' 


\section{Communication is social}

\section{Communication is about effects on people}

Table 1: Location of digital TVs and PCs

\begin{tabular}{|lcc|}
\hline & $\begin{array}{c}\text { Digital TV sets } \\
\%\end{array}$ & $\begin{array}{c}\text { PCs } \\
\%\end{array}$ \\
\hline Main living room & 92 & 18 \\
Dining room & 4 & 15 \\
Kitchen & 3 & 3 \\
Another room & 3 & 13 \\
Main bedroom & 16 & 9 \\
Another adult's bedroom & 7 & 8 \\
Child's bedroom/s & 6 & 10 \\
Study/spare room & 4 & 44 \\
\hline
\end{tabular}

and 'media' are relatively rarely used. But this may well be a mistaken abandonment of terms which have wider connotations than the term 'information'. Information is a narrower term, in effect removing any question about the reasons why people might want or need to communicate - to pass meaningful information to and from others. While in the purely technical sense, all forms of communication can be regarded as information transmission, this seems an inadequate means of grasping the essentially social underpinning of many forms of communication, and in particular the mass media. The key element is the meaning involved, the content rather than the form in which it is delivered.

Charles Horton Cooley, ${ }^{3}$ one of the founders of the social sciences, offered an analysis of 'communication' in 1912 which still holds true:

'By Communication is here meant the mechanism through which human relations exist and develop - all the symbols of the mind together with the means of conveying them through space and preserving them in time. It includes the expression of the face, attitude and gesture, the tones of the voice, words, writing, printing, railways, telegraph, telephones and whatever else may be the latest achievement on the conquest of space and time.'

In this view, the important feature of information exchange or transfer as far as people are concerned is the communication of something, of meaning - Cooley's 'mechanism'. Presenting people with informational content is not communication unless that content holds symbolic meaning for the receiver.

Changes in the means of delivery cannot be taken as representing equivalent changes in the meanings of what is delivered. It can, therefore, be argued that the process of communication is largely independent of the medium of delivery. This is not to deny that the speed, ease or effectiveness of communication are related to the choice of medium used, but it also serves to remind us that much of what we experience via the media is designed to have an effect - to communicate something to someone at one or other level. Without this approach, it is difficult to understand what other processes might lead, for example, to the existence of millions of individual personal websites on the web. Or, equally, to explain why, despite the growth of channel capacity and rival 
technologies, millions continue to watch the same TV programmes rather than drift away to different, readily available, alternatives.

One key role of the media appears to be to serve pre-existing needs and/or tastes. The clear successes of some of the newer media (or newer applications of older media) may reflect the meeting of these needs in a more effective manner than older delivery technologies: for example, e-mail and personal web pages replacing personal ads (print) or penfriends (post). This broad conclusion is supported by the findings of two large-scale studies of the uptake of technology — one on the telephone in the late 19th century, and the other on the recent boom in electronic communications systems.

'The telephone did not radically alter American ways of life; rather, Americans used it to more vigorously pursue their characteristic ways of life. 4

'Electronic communication is the number one online activity, and thus one of the most popular leisure and business activities in the United States: given that ... 62.5 per cent of all Americans send e-mail or instant messages. 5

\section{Technology's 'fit' into everyday life}

The key point to be drawn here is that the process of technological adoption relies mainly upon the ability of a given technology - or service - to 'fit' into existing patterns of life and work and, importantly, to enhance them in one way or another. Simply developing a means to increase the flow of information is not necessarily the same as enhancing communication.

\section{Interactivity}

This line of argument applies equally to media interactivity. Indeed, the concept of interactivity is central to the discussion over what differentiates 'new' media from 'old' media. Rarely, though, is this term defined in any rigorous way; rather it seems to mean many things to many people.

At the most basic level, almost everything is interactive: a door can be considered interactive in the sense that it will move when pushed. Conversely, at the most complex level, the term encompasses additional levels of meaning which relate to an exchange of information (for want of a better term - it could, for example, be argued that a physical fight between two people is extremely interactive, but not generally an act of much communicative content) and the impact of this exchange on the next stages of the exchange itself. Certainly, media can be broadly categorised into one-way media and two-way media, based on the type of interaction involved. ${ }^{6}$

- One-way media: response is called forth (but does not necessarily result in communication with the sender).

- Two-way media: response is called for (in order for the communication act to continue). 


\section{The need for evidence}

\section{New technologies account for relatively little time use}

On this basis, it can be argued that there is an urgent need to apply this type of classification to the new forms of communication. It is simply not sufficient to say that new media are different from old because they are 'interactive'. Indeed, it can be argued that the most 'interactive' medium remains the 'old' telephone (fixed or mobile) in terms of direct human-tohuman communication. In contrast, sending e-mails or text messages is not the same as talking directly with someone. Nor, arguably, is looking for a specific piece of information on a searchable database any more interactive than using a reference book. These are quite different levels of interactivity.

But knowing that people have technologies available is not the same as understanding how and why people are using them. What is needed is reliable evidence about how interactive media and messages are being adopted and, just as important, how they are actually being used by consumers. Despite the well-established levels of uptake of a range of recent technologies and associated services, there has been relatively little material which offers both reliable and generally available evidence concerning the ways in which digital media and their concomitant interactive potential are in fact being used by the UK public. This was part of the underlying rationale for the futura.com study.

The 2003 futura.com wave, as in earlier years, also assessed time use, allowing further detailed insights into how new and old communications fit into people's lives. The survey asked about both weekdays and weekend days, and covered a range of activities. Figure 3 shows the differences in time allocation between a weekday and a weekend day for a range of different activities. (The questionnaire asked respondents to estimate how might time they spent on each of a range of activities to the nearest half-hour for a typical weekday and typical weekend day. It should be noted that more than one activity can, of course, take place at one time, eg reading the paper and watching TV, or talking and listening to music.) What is clear is that those activities which are a direct result of technological innovation - such as using a PC, surfing the internet, e-mailing, texting, playing computer games or using iTV — take up a relatively small proportion of the overall time budget. At weekends there are some relatively minor shifts in time allocation (apart from workrelated uses).

The data shown in Figure 3 relate to all adults, and therefore must be expected to conceal some major differences in relative patterns of time use when examined in terms of demographics or availability of new technologies.

But looking at one of the potentially most 'extreme' contrasts - that between viewers at the weekend who had iTV available and those who only had terrestrial TV - it is clear that while there are indeed variations in the two patterns of time use, these are not particularly dramatic (Figure 4). What is notable, though, is the relatively small amount of time - for most this was under 60 minutes per weekend day — that people with iTV actually spend using interactive TV services compared with other equally or more widely available interactive digital technologies such as the internet, home PC, e-mail and texting. At the same time, though, people 


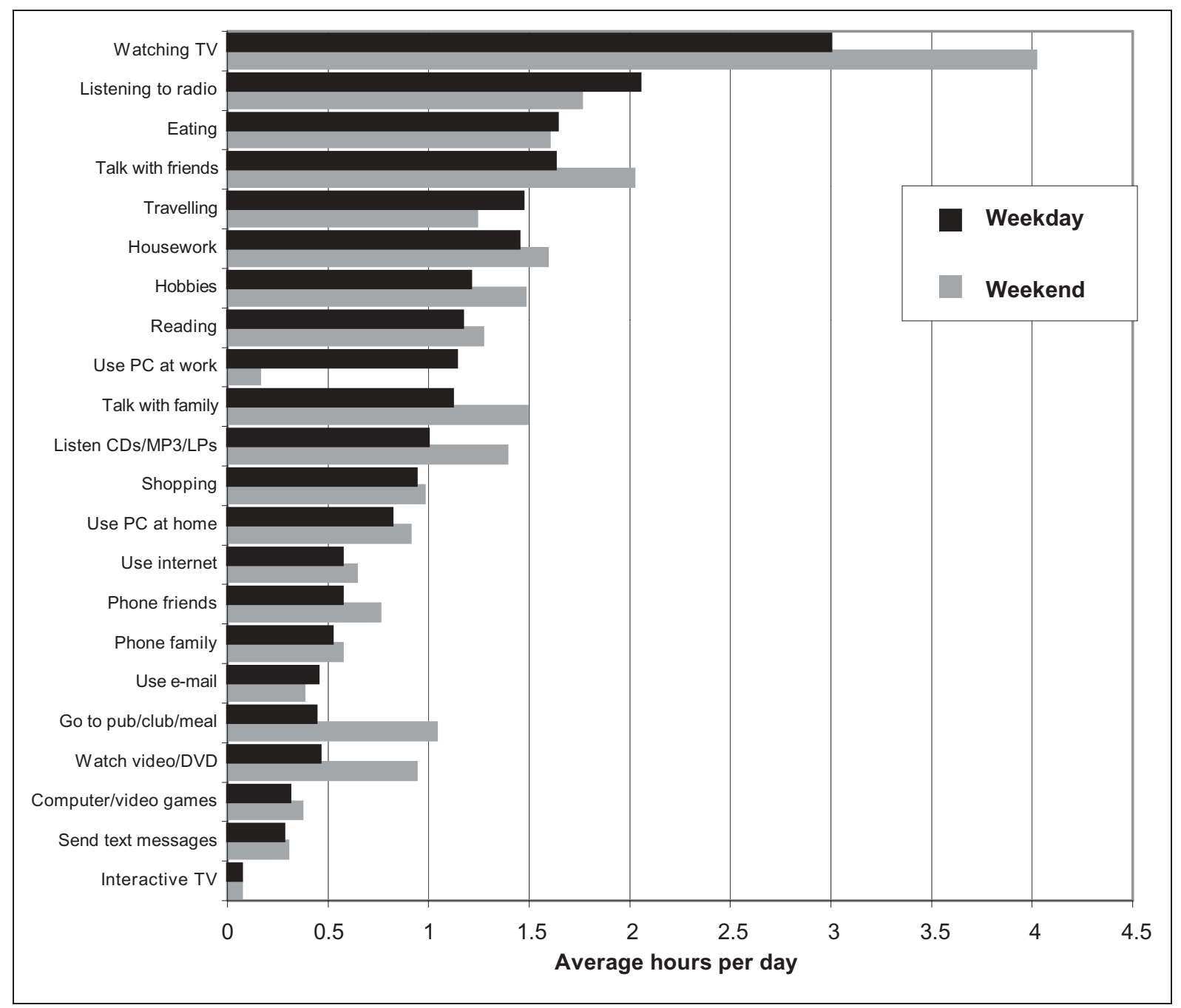

Figure 3: Average time spent on activities - all adults

with iTV spend more time watching TV (over four hours per day on average) than do terrestrial-only viewers.

\section{Television and interactivity}

Television may not, however, be the ideal route for delivering interactive services in terms of volume of use. For a start, the medium has a central social function through offering shared experiences for many, and is, together with cinema and video, highly valued for this. People have television sets (usually in more than one room) because they want to watch television programmes - a truism, but one which tends to be taken for granted. And the most popular programmes are those which show the social side of life, such as drama, soaps, comedy and 'reality' shows. This social role is not particularly compatible with the range of existing or potential interactive services, though. Some of these are implicitly designed for one-to-one or single use: either through the ways the services 


\section{Svennevig}

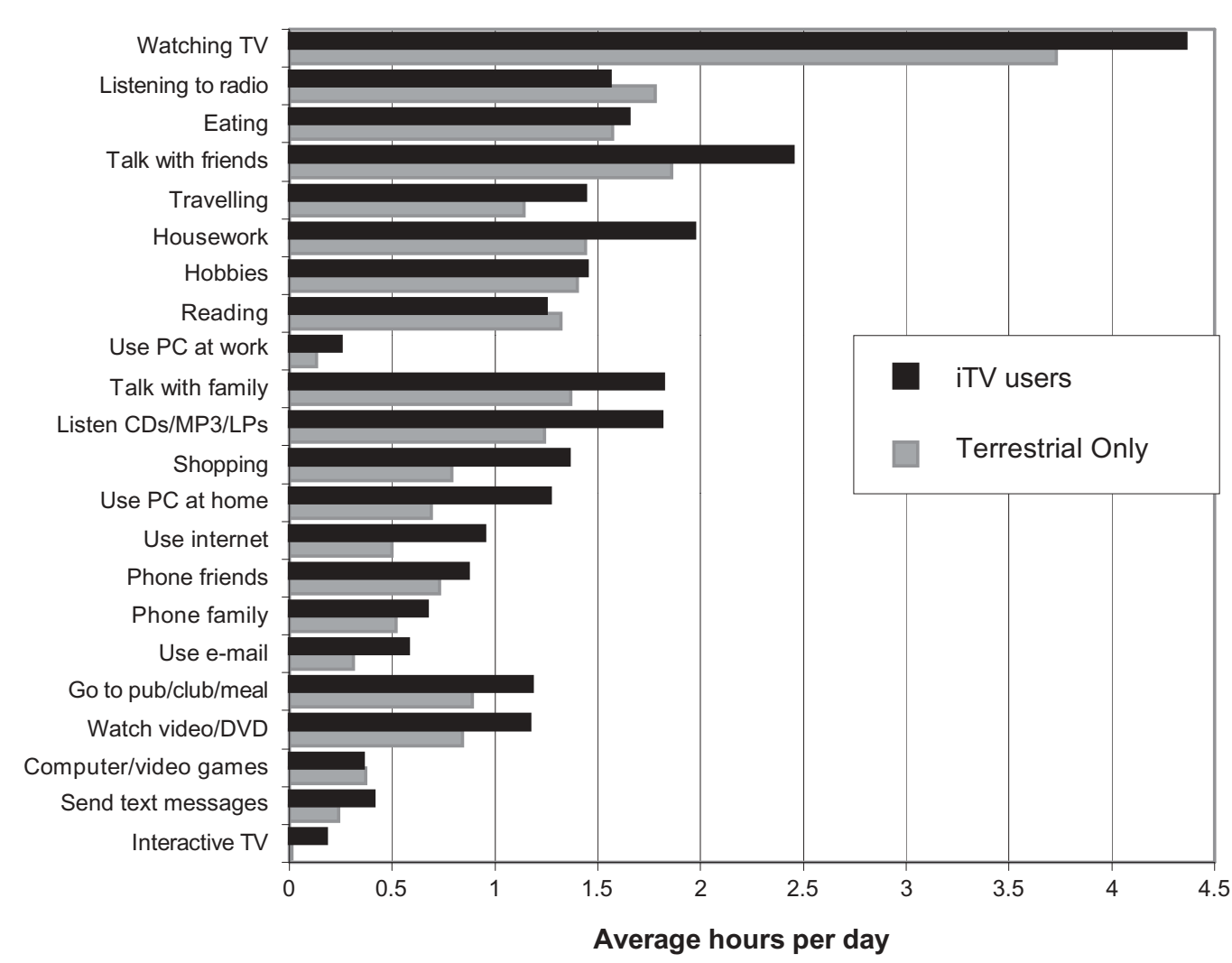

Figure 4: Interactive TV users: Time use

are set out on-screen or through the products promoted. Some evidence of this can be seen in the comparison between the ways that iTV services and PC-delivered services are used.

The 2003 futura.com survey asked all those in the sample who had iTV available at home about their use of 12 of the main available interactive TV services. The results show clearly that the most frequently used services are those directly linked to the television medium itself — the interactive services available via iTV such as the IPG (interactive programme guide) are used as enhancements to TV rather than as a means of branching out or away from the established ways of using the medium. To an extent, of course, the level of use of these features depends upon the level of supply and the quality of that supply. Where programmes do offer additional material or camera positions, these would seem to be popular — but these additions are relatively rarely offered outside of sports and reality TV shows. Clearly there are some possible interactive marketing opportunities to be found in this particular domain of 'enhanced' television programming.

The survey also included a further set of 32 different services and functions which could be accessed via the internet and which, therefore, could also be accessed by most people via iTV. For each service or function, internet users were asked whether they personally ever used it 
iTV has few advantages over the PC and which internet access route they used - PC, iTV or mobile phone (Figure 5). The mobile phone results are not discussed further here suffice it to say few, if any, of the sample reported using this route for any of the services asked about.

Figure 6 shows results for a selection of the services included in the survey. The data are based on those people who had iTV and used the internet. As with the previous example (Figure 5), there are clear and consistent differences in which services are felt by iTV users to be appropriate for each access route. Indeed, for all 32 services asked about, there were only two or three where iTV was seen to be as appropriate an access route as the PC. And these few were all related directly to television itself: voting in programmes, background information on TV programmes and links to radio and audio web channels.

It is reasonably clear from these examples from the futura.com data that there is little evidence that interactive services delivered via TV have any immediate advantage over those delivered via internet to the PC.

There are of course qualifications to be made, though. Figures 5 and 6 support the interpretation that the medium itself may determine what forms of interactive material are used: so TV encourages TV-related interactive uses. In some ways, this is hardly surprising: television - in marked contrast to the PC - offers ready-made content without the user having to contribute to its construction, in well-established branded packaging (channels). Television entertains.

Nevertheless, it is also the case that people are acquiring new technologies and services at an impressively fast rate. While it may not be

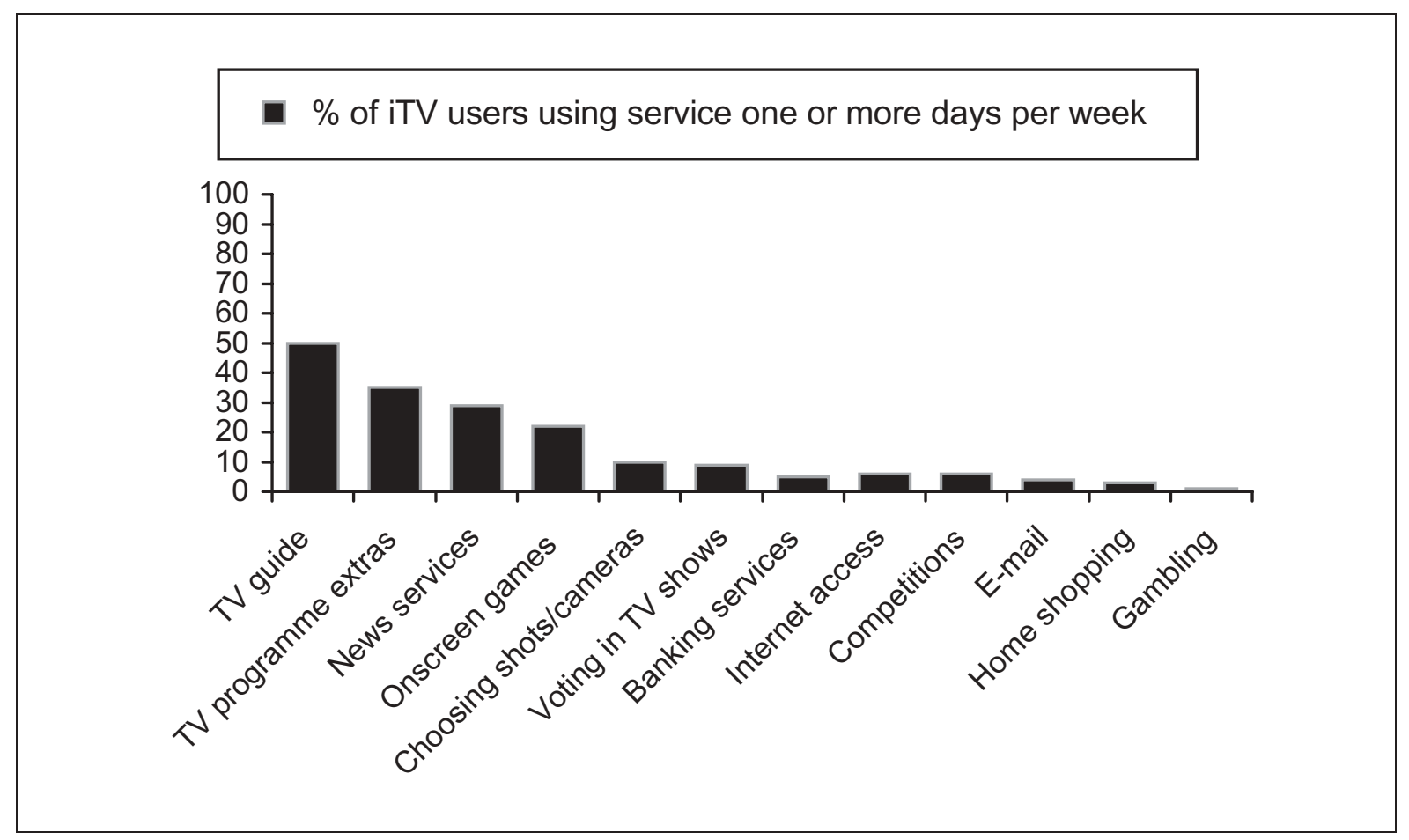

Figure 5: Uses of interactive TV 


\section{Svennevig}

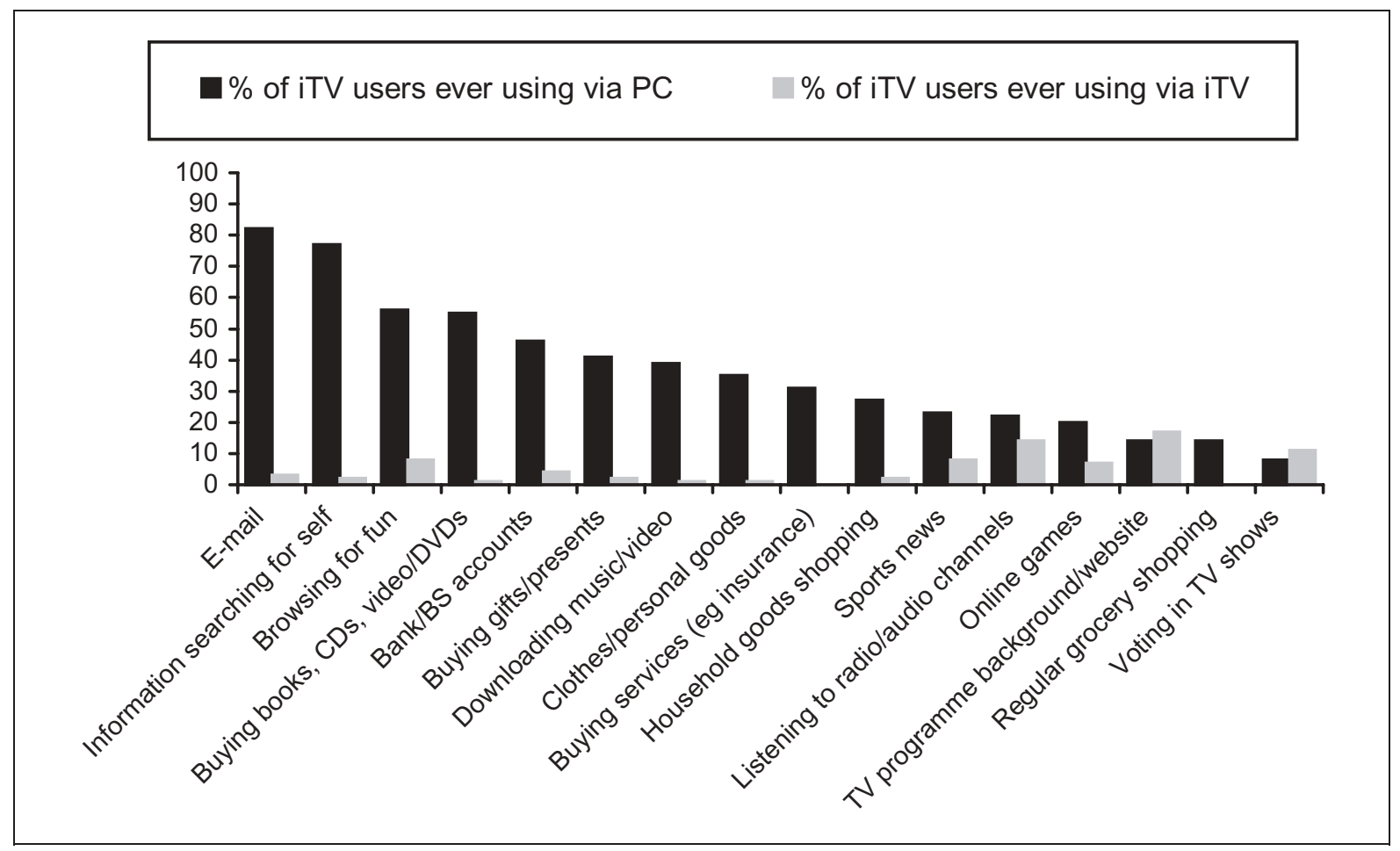

Figure 6: Uses of the internet

\section{Production skills are still important}

the case that the old destroys the new, equally there is a case to be made that there have to be some changes in behaviour, tastes and media preferences which must ensue from this expansion of choice. One such change is seen in a continuing fragmentation of time use and media use as more media options become available to consumers. As a possible guide to future changes, data have recently been made available by Nielsen NetRatings ${ }^{7}$ on the accesses made to Euro 2004 football sites by UK internet users. Intriguingly - and possibly significantly - the most visited websites during the first full week (11-18 June) were those run by broadcasters: the BBC and Sky Sports. In this context, the huge popularity of $\mathrm{BBC}$ Online is also worth bearing in mind. One tentative interpretation of these data is that broadcasters are better at assembling and presenting web material than others are; they are in the business of producing and then disseminating meaningful content. They also, of course, have access to privileged material.

\section{A generation gap?}

Another potential source of genuine change is generational; younger people who have grown up with a particular media mix may learn and retain different ways of using media and communication technologies. Looking backwards 50 years or so, there are several possible 'media generations': those who grew up with a few national and universal TV and radio channels; those who grew up with early satellite or cable and commercial radio services; and those who have grown up in the age of 


\section{Younger people are less attached to TV}

emerging digital technologies — PCs, mobiles, the internet, broadband delivery, vastly increased numbers of TV and radio channels and iTV. In the latter group are today's children, who do not feature in the futura.com sample. But teenagers and young adults are present in the sample, and provide a means of looking at how orientations towards the various media might be changing as a result of exposure to and the increasing commonplace nature of the new.

A range of questions about overall attitudes towards 'old' and 'new' technologies have been asked regularly on futura.com. Two of these are attitudinal statements which provide particularly useful measures in terms of looking at generational differences: 'I'd be lost without the internet' and 'I'd be lost without TV'. Putting these two together provides a useful guide as to how future attitudes might lie. Figure 7 shows how younger people are distinctly different from older people in terms of their attachment to TV as a medium; from age 34 and down, the overall tendency seen is for TV and the internet to be equally valued. Among teenagers, though, the internet appears to have a greater appeal than television. In contrast, those aged 35 and over show overall loyalty to television, especially among the over-55s, where the internet has few advocates.

This can be taken as evidence that there is a media use shift taking place: it is still too early to say how profound it might be, and whether it will sustain as the various media - both old and new - in their own turn evolve in terms of services and content offered in order to meet their new audiences' and users' expectations.

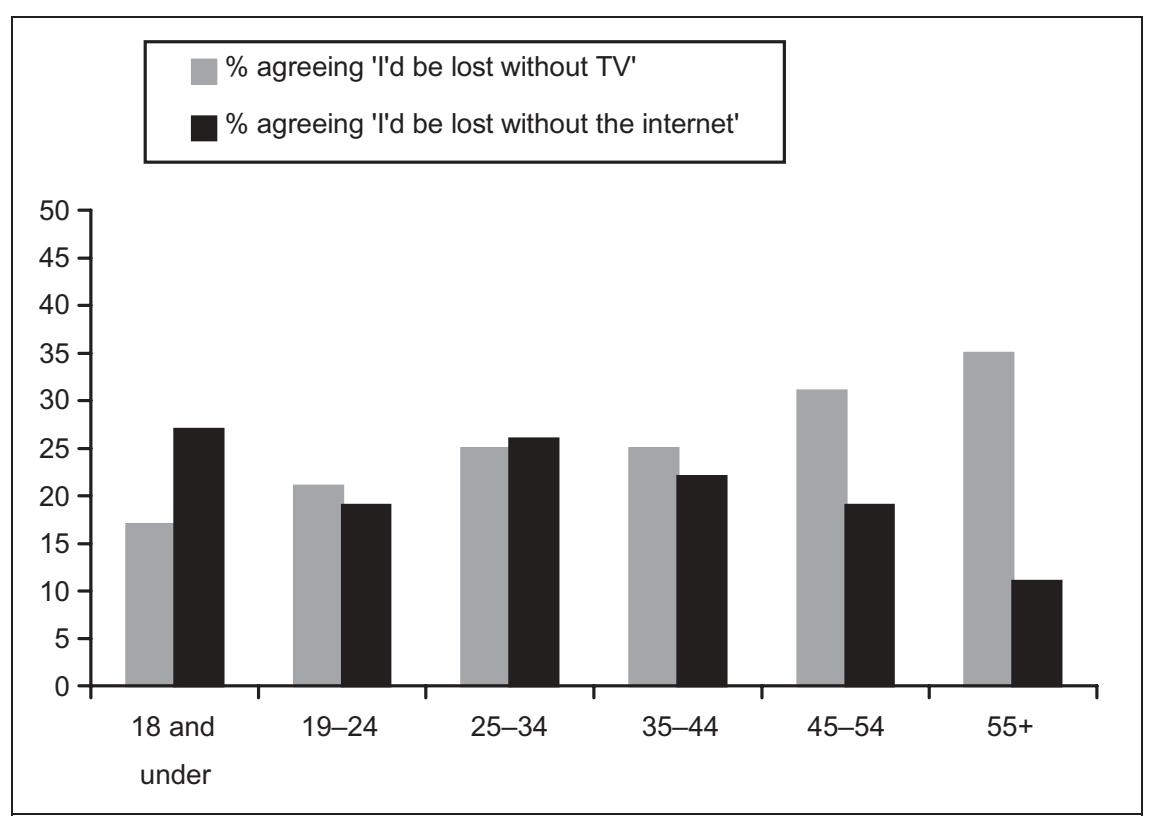

Figure 7: Attitudes to old and new media 


\section{TV survives because of what it is}

\section{People view content, not media}

\section{Conclusions}

Looked at from a suitable distance, the survival of TV is an interesting phenomenon. Despite the arrival of hi-fi, CDs, PCs, the internet, DVDs and mobile telephony, watching TV programmes still forms the main focus of leisure-time activity for the bulk of those who have a TV set. Certainly, TV time can be eroded by other technologies, especially video (albeit a 'close cousin' of TV), as well as by the PC and the internet. But TV to date is surviving such competition well: taking the evolutionary parallel of a great survivor against all the odds, it is the electronic equivalent of the rat. A key question which is rarely asked is 'Why has the TV set not evolved or been displaced?' Equally, the question can be turned around: 'Why have people remained so loyal to TV despite the other offers available?'

The answer to either question is not easy to pin down. At one level, one could argue that TV survives because of what it is - a means of being entertained, educated and informed which is low cost, requires no technical knowledge to work it, involves no need to be able to read or write or type, nor any need for training courses or a CD ROM which comes with the set to explain how to use it and no need to make any real effort other than to get into a seat.

At another level, there is the content offered. Most popular TV programming is about people - drama, documentary, entertainment, soaps, chat shows, 'soft' news stories and so on. People like seeing people, even if mediated by the TV screen, and enjoy being brought into their lives, experiences and emotions: all human life is there. Linked to this, and lying at the heart of the TV experience, there is the often overlooked art of TV production: programme makers actively seek to attract and to engage their audiences - non-simultaneous interactivity of a distinct and long-established type. Equally, since the first days of TV research has identified the powerful 'window on the world' role of the medium. And there is another functionalist or econometric level of explanation, namely that people spend more time watching TV than doing most other available activities because they enjoy it more or value it more. Whatever the detailed benefits of viewing might be, they are observably powerful and widespread, and appear at present to be relatively immune to being displaced to any great degree.

'Despite the apparent simplicity of much television programming and the taken-for-granted nature of the [TV] set's presence in people's homes, the viewing culture is, in fact, a quite complex social world. In attempting to determine the meaning of viewing per se, there are numerous factors to consider, including the ways in which the different symbolic forms of programming work. ${ }^{8}$

Also, the discussion and debate about what new technologies might mean, and how they can be used in the marketing context, do need to be far more firmly grounded in what is already well known and researched about how people use the various media in their lives. The new needs to be set into the context of the old and the current, and cannot be treated as stand-alones. 
'People construct their own media systems. From the many alternatives available to them - newspapers, radio, television, VCR and movies, compact discs and stereos, books, magazines, newsletters and pamphlets and so on - people put together their particular combinations of media and their particular relationships with these media. ${ }^{9}$

The evidence to date does not really indicate that this general conclusion (dating from 1989) has lost its appropriateness: the patterns of relative media use may well be shifting just as they always have in response to social and technological change, but the psychological and social underpinnings that influence media use and the mechanisms of appeal and impact cannot be assumed to be shifting. Thus, while there may well be a generation-in-waiting who are less attached to the 'traditional' media and who are at home with interactive media, there is still no good reason to suppose that the driving forces that create demand for media and technology, and for entertainment and news and so on and including those forces that govern purchase decisions - are themselves changing in any significant ways.

\section{References}

1. Castells, M. (1996) The Rise of the Network Society, Blackwell, London.

2. Svennevig, M. (2000) 'Needs, not nerds: Researching technological change, International Journal of Advertising, Vol. 19, No. 8, pp. 645-664.

3. Cooley, C. H. (1909) Social Organisation: A Study of the Larger Mind, Charles Scribner and Sons, New York, p. 61.

4. Fischer, C. S. (1992) America Calling: A Social History of the Telephone to 1940, University of California Press, Berkeley p. 5.

5. UCLA Centre for Communication Policy (2003) The UCLA Internet Report - Surveying the Digital Future, Year Three, UCLA, Los Angeles, p. 54.

6. Hugill, P. J. (1999) Global Communications Since 1844, Johns Hopkins University Press, Baltimore, adapted from p. 4.

7. Nielsen NetRatings (2004) 'Football comes home on the internet', 4 July, available at http://nielsen_netratings.com.

8. Lembo, R. (2000) Thinking Through Television, Cambridge University Press, Cambridge, p. 104.

9. DeFleur, M. L. and Ball-Rokeach, S. (1989) Theories of Mass Communication, 5th edn, Longman, London, p. 309.

10. Svennevig, ref. 2 above.

\section{Appendix - The futura.com project}

The futura.com study is designed around a longitudinal panel, initially established in late 1996. Details of the project can found in Svennevig. ${ }^{10}$ The project tracks public opinion and use of a range of existing media and emerging digital technologies, as well as monitoring social activities and issues.

The project is based around an initial Great Britain (GB) representative panel of 6,500 households, recruited in late 1996. As with all panels, this initial sample size has declined over the years for a variety of reasons. The original sample was boosted in 2002 using volunteers from NOP Omnibus survey respondents, selected to ensure that the overall sample 
composition remained representative in demographic terms. Each household member is sent self-completion questionnaires approximately every 12 months. In the most recent wave of research (January-April 2003), a sample of 1,225 adults was achieved. The panel is maintained by, and postal surveys run by, NOP for the University of Leeds. The data shown have been weighted to match the GB population in terms of demographics. Each wave of futura results is compared, wherever possible, with independent sources of information to ensure that the panellists' claimed ownership and use of technology and their opinions on issues are representative. To date, there are no indications that the panel is not broadly representative of UK adult opinion.

The study has been funded by a range of commercial supporters (Mindshare, Unilever, the Independent Television Commission, Carlton TV, BBC, ntl, Procter and Gamble, Flextech, Eastern Counties News, Royal Mail, Guinness). From the outset, the guiding principle underlying futura.com has been that of context: the impact of any external change be it technological, social, political, physical - has to be placed into and interpreted within a social context.

Accordingly, the questionnaires sent to the futura panellists have covered a very wide range of topics and issues; some repeated over time, others on an ad hoc basis. Examples of the range of topics covered since 1996 are:

— time use over a range of social and individual activities, including new media

- attitudes towards innovation in technology and in other areas such as genetically modified foods

- feelings of national and regional identity

- beliefs in science and in the paranormal

- specific uses of the internet and iTV services

- issues of privacy and protection of the young

- media coverage of war and death.

The core of the questions asked in each survey wave have related to technology and media uptake and use, to tastes and preferences in media content and interest in and acquisition of a wide range of emergent and established communications technologies and applications. 